\title{
Similarity transformation technique in equal-time quantized theories
}

\section{Axel WEBER*}

Instituto de Física y Matemáticas, Universidad Michoacana de San Nicolás de Hidalgo, Edificio C-3, Ciudad Universitaria, A.P. 2-82, 58040 Morelia, Michoacán, Mexico

E-mail: axel@ifm.umich.mx

\begin{abstract}
We present the application of similarity transformations of field theoretic Hamiltonians in equaltime quantized theories to the calculation of relativistic bound states. The similarity transformations used can be derived from a generalization of the Gell-Mann-Low theorem. The muonium spectrum obtained in this approach for the relativistic value $\alpha=0.3$ of the fine structure constant is compared with corresponding calculations in light-front quantization.
\end{abstract}

PACS: 11.10.St, 03.65.Ge, 11.10.Ef

LIGHT CONE 2008 Relativistic Nuclear and Particle Physics

July 7-11, 2008

Mulhouse, France

\footnotetext{
* Speaker.
} 
Similarity transformations have been used in light-front quantized theories for quite some time now [1], in particular for the description of relativistic bound states. The general idea is to reduce the size of the subspace of Fock space that is necessary for the description of the bound state at the expense of a more complicated Hamiltonian. One very clear-cut formulation of such an approach is in terms of $N$-particle Fock space sectors [2]. While the main motivation for the similarity transformation technique is its eventual application to QCD bound states, i.e., hadrons, the approach has been tested for Yukawa theory [3] and QED [4, 5, 6].

These applications have evidenced some problems of the approach, notably an unphysical UV cutoff dependence [3,5]. In this contribution, we will apply a similar approach to equal-time quantized theories, in particular to QED. In addition to obtaining quite promising results, we hope to shed some light on the problems that appear in the context of light-front quantized theories.

In equal-time quantized theories, a similarity transformation is naturally induced by a generalization of the Gell-Mann-Low theorem [7]. For the determination of relativistic bound states, we apply the theorem to the subspace of Fock space that contains all states of the constituents as free particles. For concreteness, we will consider states of one electron and one antimuon, although we will allow the mass of the "antimuon" to take any value. As in the earlier applications of the same formalism to the Wick-Cutkosky model and Yukawa theory [8, 9], the effective Hamiltonian generated by the generalized Gell-Mann-Low theorem contains the relativistic kinetic energies of the constituents and an effective potential. As for the kinetic energies, the corrections to the "bare" values from the expectation value of the free part of the Hamiltonian are expected to lead to mass renormalizations identical to the ones in covariant formulations, from general arguments that were made explicit in the case of Yukawa theory [9]. In order to identify all-order contributions to the vacuum energy, the kinetic energies, and the interaction of the constituents, a diagrammatical representation is helpful (cf. Ref. [8]).

As for the effective potential, the matrix elements in Coulomb gauge read to lowest nontrivial order in a perturbative expansion,

$$
\begin{aligned}
\left\langle\mathbf{p}_{A}, r ; \mathbf{p}_{B}, s\left|V_{\mathrm{eff}}\right| \mathbf{p}_{A}^{\prime}, r^{\prime} ; \mathbf{p}_{B}^{\prime}, s^{\prime}\right\rangle= & -\frac{e^{2}}{\sqrt{2 E_{\mathbf{p}_{A}}^{A} 2 E_{\mathbf{p}_{B}}^{B} 2 E_{\mathbf{p}_{A}^{\prime}}^{A} 2 E_{\mathbf{p}_{B}^{\prime}}^{B}}} \\
\times & {\left[\frac{1}{\left(\mathbf{p}_{A}-\mathbf{p}_{A}^{\prime}\right)^{2}}\left[\bar{u}_{A}\left(\mathbf{p}_{A}, r\right) \gamma^{0} u_{A}\left(\mathbf{p}_{A}^{\prime}, r^{\prime}\right)\right]\left[\bar{u}_{B}\left(\mathbf{p}_{B}, s\right) \gamma^{0} u_{B}\left(\mathbf{p}_{B}^{\prime}, s^{\prime}\right)\right]\right.} \\
& -\frac{1}{2\left|\mathbf{p}_{A}-\mathbf{p}_{A}^{\prime}\right|}\left(\frac{1}{E_{\mathbf{p}_{A}}^{A}+\left|\mathbf{p}_{A}-\mathbf{p}_{A}^{\prime}\right|-E_{\mathbf{p}_{A}^{\prime}}^{A}}+\frac{1}{E_{\mathbf{p}_{B}}^{B}+\left|\mathbf{p}_{B}-\mathbf{p}_{B}^{\prime}\right|-E_{\mathbf{p}_{B}^{\prime}}^{B}}\right) \\
& \times\left[\bar{u}_{A}\left(\mathbf{p}_{A}, r\right) \gamma^{i} u_{A}\left(\mathbf{p}_{A}^{\prime}, r^{\prime}\right)\right]\left(\sum_{\lambda=1}^{2} \varepsilon_{i}^{(\lambda)}\left(\mathbf{p}_{A}-\mathbf{p}_{A}^{\prime}\right) \varepsilon_{j}^{(\lambda) *}\left(\mathbf{p}_{A}-\mathbf{p}_{A}^{\prime}\right)\right) \\
& \left.\times\left[\bar{u}_{B}\left(\mathbf{p}_{B}, s\right) \gamma^{j} u_{B}\left(\mathbf{p}_{B}^{\prime}, s^{\prime}\right)\right]\right](2 \pi)^{3} \delta\left(\mathbf{p}_{A}+\mathbf{p}_{B}-\mathbf{p}_{A}^{\prime}-\mathbf{p}_{B}^{\prime}\right) .
\end{aligned}
$$

Here, $\left|\mathbf{p}_{A}, r ; \mathbf{p}_{B}, s\right\rangle$ symbolizes the state of an electron with 3-momentum $\mathbf{p}_{A}$ and spin orientation $r$ (in a spinor basis yet to be specified) and an antimuon with 3-momentum $\mathbf{p}_{B}$ and spin orientation $s$. We use the shorthands $E_{\mathbf{p}_{A}}^{A}=\left(m_{A}^{2}+\mathbf{p}_{A}^{2}\right)^{1 / 2}$ and $E_{\mathbf{p}_{B}}^{B}=\left(m_{B}^{2}+\mathbf{p}_{B}^{2}\right)^{1 / 2}$ for the kinetic energies. For 
convenience, we have introduced the charge-conjugate Dirac spinors $u_{B}\left(\mathbf{p}_{B}, s\right)$ for the antimuon, while $u_{A}\left(\mathbf{p}_{A}, s\right)$ represent the electron spinors. The spatially transverse photon polarization vectors $\varepsilon_{i}^{(\lambda)}(\mathbf{k})$ satisfy the relation $\sum_{\lambda=1}^{2} \varepsilon_{i}^{(\lambda)}(\mathbf{k}) \varepsilon_{j}^{(\lambda) *}(\mathbf{k})=\delta_{i j}^{\mathrm{tr}}(\mathbf{k}) \equiv \delta_{i j}-\hat{k}_{i} \hat{k}_{j}$ (where $\left.\hat{\mathbf{k}}=\mathbf{k} /|\mathbf{k}|\right)$.

The interpretation of the effective potential (1) is the following: the second line stems from the instantaneous Coulomb potential, easily identified by the momentum dependence in the denominator (the Fourier transform of the spatial Coulomb potential), and multiplied with the charge densities of the Dirac currents. The following lines are the result of transverse photon exchange, the more complicated denominators indicating a retarded interaction, and the Dirac currents being contracted with the corresponding photon polarization vectors.

The delta function in Eq. (1) shows that total 3-momentum is conserved by the effective interaction, and in the following we will consider the center-of-mass system (c.m.s.) $\mathbf{p}_{A}+\mathbf{p}_{B}=$ $\mathbf{p}_{A}^{\prime}+\mathbf{p}_{B}^{\prime}=0$. In order to simplify the diagonalization of the effective Hamiltonian, we express the Dirac spinors in terms of Pauli spinors (using the Dirac-Pauli representation) to find the effective Schrödinger equation in the c.m.s.,

$$
\begin{gathered}
\left(\sqrt{m_{A}^{2}+\mathbf{p}^{2}}+\sqrt{m_{B}^{2}+\mathbf{p}^{2}}\right) \phi(\mathbf{p})-e^{2} \int \frac{d^{3} p^{\prime}}{(2 \pi)^{3}} \sqrt{\frac{E_{\mathbf{p}}^{A}+m_{A}}{2 E_{\mathbf{p}}^{A}} \frac{E_{\mathbf{p}}^{B}+m_{B}}{2 E_{\mathbf{p}}^{B}} \frac{E_{\mathbf{p}^{\prime}}^{A}+m_{A}}{2 E_{\mathbf{p}^{\prime}}^{A}} \frac{E_{\mathbf{p}^{\prime}}^{B}+m_{B}}{2 E_{\mathbf{p}^{\prime}}^{B}}} \\
\times\left[\frac{1}{\left(\mathbf{p}-\mathbf{p}^{\prime}\right)^{2}}\left(1+\frac{\mathbf{p} \cdot \boldsymbol{\sigma}_{A}}{E_{\mathbf{p}}^{A}+m_{A}} \frac{\mathbf{p}^{\prime} \cdot \boldsymbol{\sigma}_{A}}{E_{\mathbf{p}^{\prime}}^{A}+m_{A}}\right)\left(1+\frac{\mathbf{p} \cdot \boldsymbol{\sigma}_{B}}{E_{\mathbf{p}}^{B}+m_{B}} \frac{\mathbf{p}^{\prime} \cdot \boldsymbol{\sigma}_{B}}{E_{\mathbf{p}^{\prime}}^{B}+m_{B}}\right)\right. \\
+\frac{1}{2\left|\mathbf{p}-\mathbf{p}^{\prime}\right|}\left(\frac{1}{E_{\mathbf{p}}^{A}+\left|\mathbf{p}-\mathbf{p}^{\prime}\right|-E_{\mathbf{p}^{\prime}}^{A}}+\frac{1}{E_{\mathbf{p}}^{B}+\left|\mathbf{p}-\mathbf{p}^{\prime}\right|-E_{\mathbf{p}^{\prime}}^{B}}\right)\left(\frac{\left(\mathbf{p} \cdot \boldsymbol{\sigma}_{A}\right) \sigma_{A}^{i}}{E_{\mathbf{p}}^{A}+m_{A}}+\frac{\sigma_{A}^{i}\left(\mathbf{p}^{\prime} \cdot \boldsymbol{\sigma}_{A}\right)}{E_{\mathbf{p}^{\prime}}^{A}+m_{A}}\right) \\
\left.\times \delta_{i j}^{\operatorname{tr}}\left(\mathbf{p}-\mathbf{p}^{\prime}\right)\left(\frac{\left(\mathbf{p} \cdot \boldsymbol{\sigma}_{B}\right) \sigma_{B}^{j}}{E_{\mathbf{p}}^{B}+m_{B}}+\frac{\boldsymbol{\sigma}_{B}^{j}\left(\mathbf{p}^{\prime} \cdot \boldsymbol{\sigma}_{B}\right)}{E_{\mathbf{p}^{\prime}}^{B}+m_{B}}\right)\right] \phi\left(\mathbf{p}^{\prime}\right)=E^{\prime} \phi(\mathbf{p}) .
\end{gathered}
$$

Here, $\mathbf{p}=\mathbf{p}_{A}=-\mathbf{p}_{B}$, the spinorial wave function $\phi(\mathbf{p})$ is defined as $\phi\left(\mathbf{p}_{A}\right)(2 \pi)^{3} \delta\left(\mathbf{p}_{A}+\mathbf{p}_{B}\right)=$ $\sum_{r, s}\left\langle\mathbf{p}_{A}, r ; \mathbf{p}_{B}, s \mid \phi\right\rangle\left[\chi_{r} \otimes \chi_{s}\right]$, and $\boldsymbol{\sigma}_{A}\left(\boldsymbol{\sigma}_{B}\right)$ is understood to act on the Pauli spinor $\chi_{r}\left(\chi_{s}\right)$ only. $E^{\prime}$ is the difference between the energy of the bound state and the vacuum energy. Of the full state $|\phi\rangle$ in Fock space (with zero total momentum), only its projection to one-electron-one-antimuon states $\left|\mathbf{p}_{A}, r ; \mathbf{p}_{B}, s\right\rangle$ appears. The effect of its components in other Fock space sectors is implicitly taken care of (to the order considered) by the effective potential. Equation (2) is a well-defined equation which admits a discrete spectrum of eigenvalues as can be shown by a detailed analytical investigation of the large-momentum behavior of its solutions, and by a direct numerical solution of the equation to which we now turn.

To this end, we take into account the rotational and parity invariance of Eq. (2). Eigenstates of total angular momentum $J$ can be constructed as usual by adding relative orbital angular momentum $L$ and total spin $S$. For convenience, instead of using parity itself, we will label the eigenstates by the "relative parity" $\pi^{\prime}$ defined through $(-1)^{L}=\pi^{\prime}(-1)^{J}$. Since $S=0,1$, for given $J$ the sector $\pi^{\prime}=+1$ contains the states with $L=J$ and $S=0$ or $S=1$, while for $\pi^{\prime}=-1$ we can have $L=J-1$ or $L=J+1$, with $S=1$ in both cases. In any sector $J^{\pi^{\prime}}$, the two different possible $(L S)$-states will mix, except for $J=0$ (only one state exists in each of the two sectors), for equal masses [the states of the $\left(\pi^{\prime}=+1\right)$-sector do not mix because of the additional exchange symmetry], and in 
the one-body limit where the mass of one of the constituents goes to infinity [no mixing in the $\left(\pi^{\prime}=-1\right)$-sector]. The one-body limit will be discussed more fully later on.

After explicitly carrying out the contractions of the spatial indices in the transverse photon exchange part, the formulae employed before in the solution of the effective Schrödinger equation for the case of Yukawa theory [9] can be used for the present case. We will focus here on the partial-wave decomposition which is well-known for the (Fourier transformed) Coulomb potential, and has been calculated in Ref. [9] for the $\delta_{i j}$-part of the transverse photon exchange. The partial waves of the $\hat{k}_{i} \hat{k}_{j}$-part of the transverse photon exchange have not appeared before and are given by

$$
\begin{aligned}
b_{L}\left(p, p^{\prime}\right)= & \frac{2 L+1}{2} \int_{-1}^{1} d \cos \theta P_{L}(\cos \theta) \\
& \times \frac{1}{\left(\mathbf{p}-\mathbf{p}^{\prime}\right)^{2}} \frac{1}{2\left|\mathbf{p}-\mathbf{p}^{\prime}\right|}\left(\frac{1}{E_{p}^{A}+\left|\mathbf{p}-\mathbf{p}^{\prime}\right|-E_{p^{\prime}}^{A}}+\frac{1}{E_{p}^{B}+\left|\mathbf{p}-\mathbf{p}^{\prime}\right|-E_{p^{\prime}}^{B}}\right) .
\end{aligned}
$$

The integral diverges like $\left(p-p^{\prime}\right)^{-2}$ for $p^{\prime} \rightarrow p$ which would lead to a divergence in the $p^{\prime}$-integral. These divergences are spurious and cancel in pairs in the complete expressions. However, for the numerical calculation, we have to extract the divergent parts and perform the cancellations analytically. Fortunately, the extraction of the divergencies is simple: they occur at $\cos \theta=1$, and since $P_{L}(1)=1$, we can conveniently split $P_{L}(\cos \theta)$ in $\left[1+(\cos \theta-1) P_{L}^{R}(\cos \theta)\right]$, thus defining a "reduced" Legendre polynomials $P_{L}^{R}(\cos \theta)$. The divergent parts in Eq. (3) originating from the 1 in this decomposition can be analytically cancelled in pairs, leaving a finite contribution. The remainder of the integral is logarithmically divergent for $p^{\prime} \rightarrow p$ (as are the other partial waves), and the following $p^{\prime}$-integration is convergent.

The rest of the numerical solution of Eq. (2) proceeds in strict analogy with the Yukawa case [9]. The results for the lowest energy eigenvalues thus obtained are plotted in Figs. 1 and 2 for equal constituent masses and fine structure constants $\alpha \leq 0.45$. The binding energies are normalized to $\mu \alpha^{2}, \mu$ being the reduced mass, so that the comparison with the nonrelativistic energy eigenvalues $\mu \alpha^{2} / 2 n^{2}$ is immediate.

We find that for values $\alpha<0.1$, the energy levels are dominated by the nonrelativistic values plus the leading relativistic corrections (the leading-order fine and hyperfine structure) of order $\mu \alpha^{4}$, both in our numerical results and in the perturbative calculations of bound-state QED. In this region of small coupling constants, the numerical results are in good agreement with the perturbative calculations, apparently only limited by the numerical precision. For larger values $\alpha>0.1$, higher perturbative orders become important and our numerical results deviate in some cases strongly from the lowest-order perturbative predictions.

In Table 1 we compare our results for $\alpha=0.3$ with two different calculations in light front quantization [5, 6] (we use the data for the Gaussian similarity function in the latter paper). In the table, we label the states by the nonrelativistic notation $n^{2 S+1} L_{J}$ and also indicate the corresponding sectors $J^{\pi^{\prime}}$. There is a clear tendency in our results towards more negative energies, i.e., stronger binding, compared to $\mathscr{O}\left(\mu \alpha^{4}\right)$-perturbation theory. The ordering of the different levels, however, is the same as in perturbation theory. We can also see that the difference to perturbation theory in the direction of stronger binding is systematically larger for $S$-states than for $P$-states, and also larger for $(J=0)$-states than for $(J=1)$-states, and smallest for the $(J=2)$-state. For the light-front results, this latter tendency is inverted; the $S_{0}$-states have even higher energies than in perturbation 


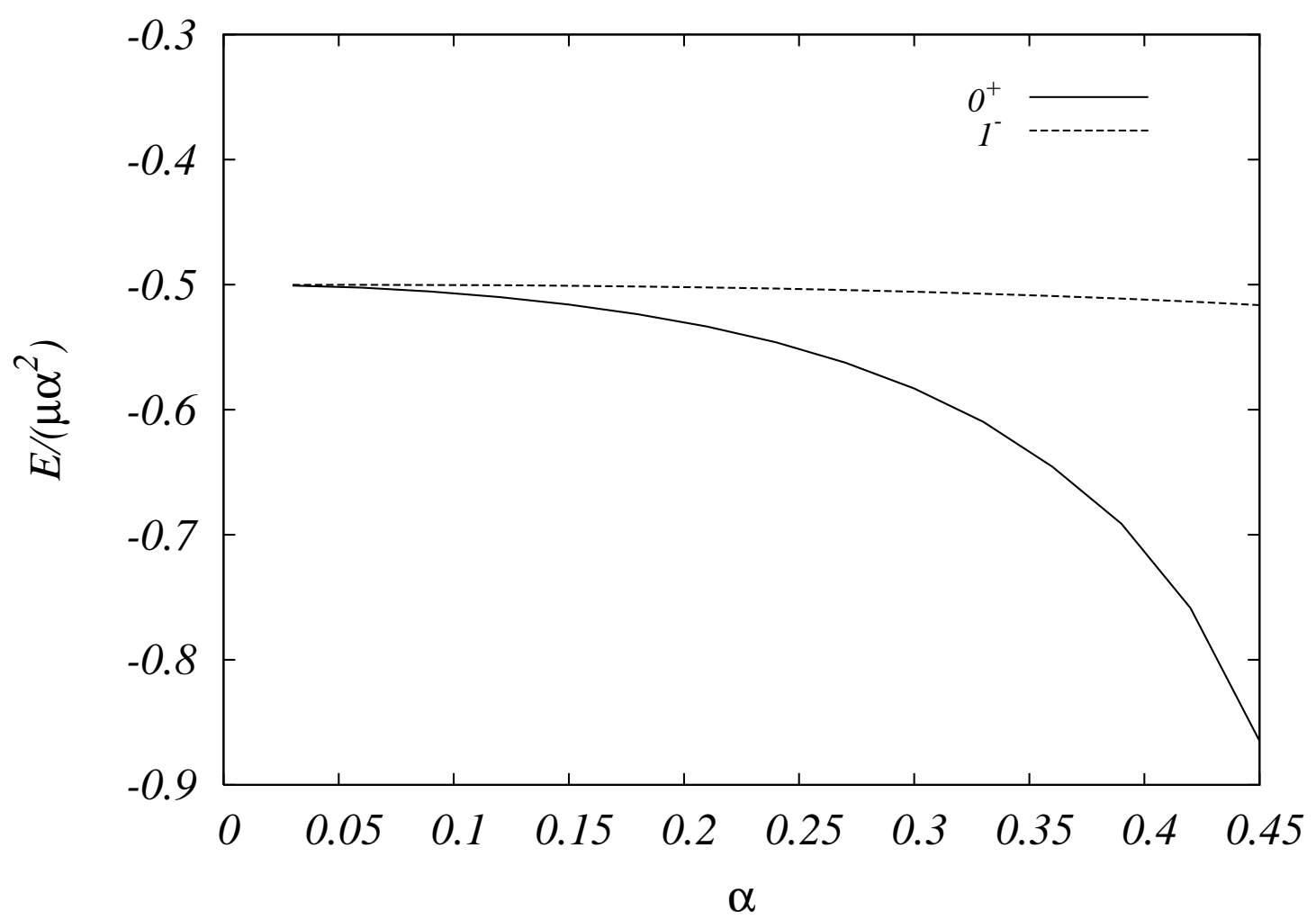

Figure 1: The binding energy $E=E^{\prime}-m_{A}-m_{B}$ as a function of the fine structure constant $\alpha=e^{2} / 4 \pi$ for the case of equal masses $m_{A}=m_{B} . E$ is normalized to $\mu \alpha^{2}$ where $\mu$ is the reduced mass $\left(\mu=m_{A} / 2\right.$ in the present case). Plotted are the lowest energy levels for $J^{\pi^{\prime}}=0^{+}$and $1^{-}$corresponding to the nonrelativistic principal quantum number $n=1$.

\begin{tabular}{ccccc}
\hline state & $\begin{array}{c}\text { perturbation } \\
\text { theory }\end{array}$ & $\begin{array}{c}\text { our } \\
\text { results }\end{array}$ & Ref. [5] & Ref. [6] \\
\hline $1^{1} S_{0}\left(0^{+}\right)$ & -0.559 & -0.583 & -0.525 & -0.551 \\
$1^{3} S_{1}\left(1^{-}\right)$ & -0.499 & -0.506 & -0.501 & -0.525 \\
$2{ }^{1} S_{0}\left(0^{+}\right)$ & -0.1343 & -0.1373 & -0.1301 & -0.1332 \\
$2{ }^{3} P_{0}\left(0^{-}\right)$ & -0.1306 & -0.1315 & -0.1335 & -0.1369 \\
$2{ }^{3} P_{1}\left(1^{+}\right)$ & -0.1278 & -0.1279 & -0.1298 & -0.1327 \\
$2{ }^{3} S_{1}\left(1^{-}\right)$ & -0.1268 & -0.1277 & -0.1269 & -0.1298 \\
$2{ }^{1} P_{1}\left(1^{+}\right)$ & -0.1268 & -0.1269 & -0.1290 & -0.1315 \\
$2{ }^{3} P_{2}\left(2^{-}\right)$ & -0.1255 & -0.1255 & -0.1277 & -0.1302 \\
\hline
\end{tabular}

Table 1: Binding energies $E / \mu \alpha^{2}$ for equal masses from perturbation theory to $\mathscr{O}\left(\mu \alpha^{4}\right)$, from our numerical results, and from Refs. [5] and [6] 


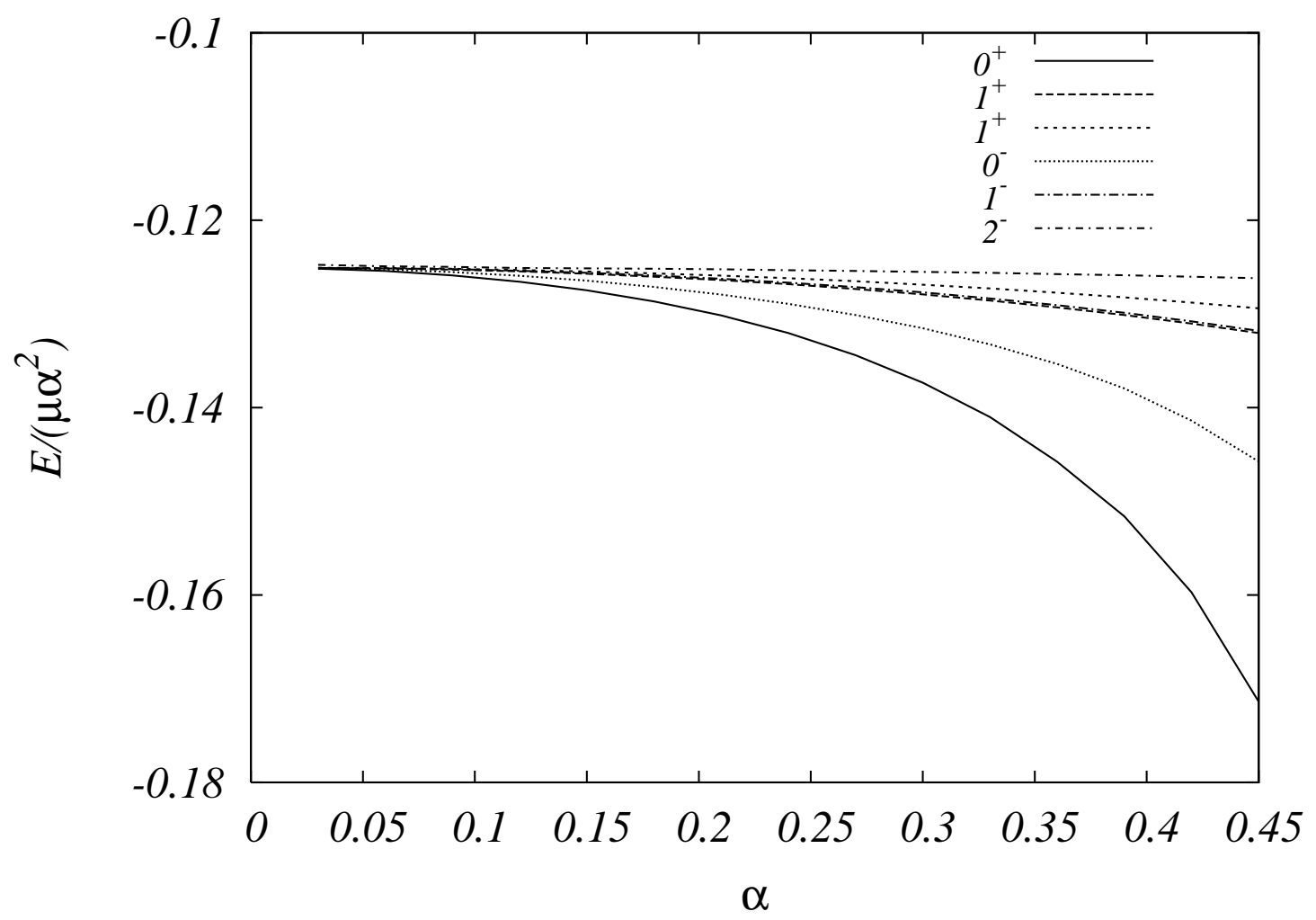

Figure 2: As Fig. 1, but for the energy levels corresponding to the nonrelativistic principal quantum number $n=2$.

theory. Both light-front calculations are qualitatively similar, only that the binding is stronger throughout in the similarity transform approach of Ref. [6]. In conclusion, our approach gives qualitatively different results from the similarity transformation technique applied to the light-front quantized theories (in the approximations presently considered). We emphasize, however, that there is an unphysical logarithmic UV cutoff dependence in the light-front results (for the cited values, the cutoff has been set equal to the constituent masses).

In order to gain additional insight into the approach presented, it is helpful to consider the one-body limit, i.e., the limit of infinite mass of the antimuon. One can show that in this limit the Schrödinger equation (2) reduces to the equation for an electron in an external potential. The contribution from transverse gluon exchange becomes suppressed and only the instantaneous Coulomb interaction remains (similarly to the nonrelativistic limit). Also, the spin of the heavy antimuon decouples from the dynamics, leading to the exact degeneracy (within numerical precision) of pairs of states in the numerical solution of Eq. (2) in this limit.

We can combine the effective Schrödinger equation for the electron wave function $\phi_{A}(\mathbf{p}, r)$ in the one-body limit with the charge conjugate equation for a positron wave function $\bar{\phi}_{A}(\mathbf{p}, r)$ to obtain the Dirac form

$$
\left(\boldsymbol{\alpha} \cdot \mathbf{p}+\beta m_{A}\right) \phi(\mathbf{p})-\int \frac{d^{3} p^{\prime}}{(2 \pi)^{3}}\left\{\Lambda_{+}^{A}(\mathbf{p}) \frac{e^{2}}{\left(\mathbf{p}-\mathbf{p}^{\prime}\right)^{2}} \Lambda_{+}^{A}\left(\mathbf{p}^{\prime}\right)\right.
$$




$$
\begin{gathered}
\left.+\Lambda_{-}^{A}(\mathbf{p}) \frac{e^{2}}{\left(\mathbf{p}-\mathbf{p}^{\prime}\right)^{2}} \Lambda_{-}^{A}\left(\mathbf{p}^{\prime}\right)\right\} \phi\left(\mathbf{p}^{\prime}\right)=E^{\prime} \phi(\mathbf{p}) \\
\text { with } \phi(\mathbf{p})=\sum_{r=1}^{2} \frac{1}{\sqrt{2 E_{\mathbf{p}}^{A}}}\left[\phi_{A}(\mathbf{p}, r) u_{A}(\mathbf{p}, r)+\bar{\phi}_{A}^{*}(-\mathbf{p}, r) v_{A}(-\mathbf{p}, r)\right],
\end{gathered}
$$

where $\Lambda_{ \pm}^{A}(\mathbf{p})$ are the usual Casimir projectors to positive and negative energy solutions. They decouple particle and antiparticle equations which distinguishes Eq. (4) from the Dirac equation. Although we thus lose the cherished covariance properties of the Dirac equation, we gain a clear one-particle interpretation of the wave function. The complete Fock state including arbitrary numbers of electron-positron pairs can also be recovered perturbatively in our approach. In an expansion around the nonrelativistic limit, the projectors do not contribute to the first corrections, and Eq. (4) reproduces the fine structure contained in the Dirac equation.

Analogously, in the case of two dynamical particles, the effective Schrödinger equation for particles $A B$ can be combined with the charge conjugate equation for two antiparticles $\overline{A B}$. The result is a reduced Salpeter equation where the instantaneous interaction is obtained from first principles via the generalized Gell-Mann-Low theorem. The first relativistic corrections to the nonrelativistic limit yield the Breit interaction and, as a consequence, the correct fine and hyperfine structure of the system. The Casimir projectors again guarantee a two-particle wave function interpretation and avoid anomalies like the Brown-Ravenhall disease.

\section{Acknowledgments}

Support by Conacyt grant 46513-F and CIC-UMSNH is gratefully acknowledged. I thank my collaborator Juan Carlos López Vieyra for performing the numerical calculations.

\section{References}

[1] St. D. Głazek and K. G. Wilson, Phys. Rev. D 49 (1994) 4214; F. Wegner, Ann. Phys. 3 (1994) 77; H.-C. Pauli, Solving gauge field theory by discretized light-cone quantization, hep-th/9704215; T. S. Walhout, Phys. Rev. D 59 (1999) 065009.

[2] E. L. Gubankova and F. Wegner, Phys. Rev. D 58 (1998) 025012.

[3] St. Głazek, A. Harindranath, S. Pinsky, J. Shigemutsu, and K. Wilson, Phys. Rev. D 47 (1993) 1599; S. J. Brodsky, H.-C. Pauli, and S. S. Pinsky, Phys. Rep. 301 (1998) 299.

[4] B. D. Jones, R. J. Perry, and St. D. Głazek, Phys. Rev. D 55 (1997) 6561.

[5] U. Trittmann and H.-C. Pauli, Quantum electrodynamics at strong couplings, hep-th/9704215.

[6] E. L. Gubankova, H.-C. Pauli, F. J. Wegner, and G. Papp, Light-cone Hamiltonian flow for positronium, hep-th/9809143.

[7] A. Weber, Bloch-Wilson Hamiltonian and a generalization of the Gell-Mann-Low theorem, in Particles and Fields - Seventh Mexican Workshop, AIP Conf. Proc. 531, AIP, New York 2000, p. 305.

[8] A. Weber and N. E. Ligterink, Phys. Rev. D 65 (2002) 025009.

[9] A. Weber and N. E. Ligterink, Bound states in Yukawa theory, hep-ph / 0506123. 